\title{
Clinical Outcome between Ticagrelor versus Clopidogrel in Patients with Acute Coronary Syndrome and Diabetes
}

\author{
Peixun He, ${ }^{1}$ Xiaolin Luo, ${ }^{1}$ Jiabei Li, ${ }^{1}$ Yi Li, ${ }^{2}$ Xiaozeng Wang, ${ }^{2}$ Lan Huang, ${ }^{1}$ Jun Jin $\mathbb{D}^{1},{ }^{1}$ \\ and Yaling Han $\mathbb{1}^{2}$ \\ ${ }^{1}$ Department of Cardiology, The Second Affiliated Hospital, Army Medical University, Chongqing, China \\ ${ }^{2}$ Department of Cardiology, General Hospital of Northern Theater Command, Shenyang, China \\ Correspondence should be addressed to Jun Jin; jjin918@163.com and Yaling Han; hanyaling@263.net
}

Received 18 January 2021; Revised 7 July 2021; Accepted 11 September 2021; Published 15 October 2021

Academic Editor: Prasanth Puthanveetil

Copyright (c) 2021 Peixun He et al. This is an open access article distributed under the Creative Commons Attribution License, which permits unrestricted use, distribution, and reproduction in any medium, provided the original work is properly cited.

\begin{abstract}
Background. The increased thrombotic risk in patients with acute coronary syndrome (ACS) and diabetes highlights the need for adequate antithrombotic protection. We aimed to compare the 6-month clinical outcomes between ticagrelor and clopidogrel in patients with ACS and diabetes. Methods and Results. The study was a single-center, prospective, randomized, open-label, blinded endpoint, and controlled registry trial. A total of 270 ACS patients with diabetes were randomly assigned in a $1: 1$ ratio to either the ticagrelor group or the clopidogrel group. Follow-up was performed for 6 months, and the data on efficacy outcomes and bleeding events were collected. At 6 months, complete follow-up data were available for 266 (98.5\%) of 270 patients, and 4 were lost to follow-up. There was no significant difference in the survival rate of the effective endpoints between the ticagrelor group $(n=133)$ and the clopidogrel group $(n=133)$ (HR $0.83,95 \%$ CI $0.44-1.56, p=0.561)$, but the incidence of bleeding events in the ticagrelor group was higher than that in the clopidogrel group (HR 1.76, 95\% CI 1.00-3.10, $p=0.049$ ). Conclusion. Ticagrelor did not improve the composite of nonfatal MI, target vessel revascularization, rehospitalization, stroke, and death from any cause; however, it significantly increased the incidence of bleeding events defined by the Bleeding Academic Research Consortium (BARC) criteria in Chinese patients with ACS and diabetes during the 6-month follow-up compared with clopidogrel.
\end{abstract}

\section{Introduction}

Acute coronary syndrome (ACS) is one of the major lethal and disabling diseases that affect millions of people worldwide [1]. Following atherosclerotic plaque rupture inside a coronary artery, the initiation of thrombus formation by platelet activation is a major element [2]; ergo, antiplatelet therapy is a landmark treatment strategy for ACS. In China, up to $37 \%$ of patients presenting with ACS suffer from diabetes [3]. Among ACS patients, diabetic status was associated with more elements of the ischemic cardiovascular profile [4]; this may be partly related to abnormal platelet function leading to platelet hyperreactivity. Previous studies in patients with ACS and diabetes showed a 1.8-fold increase in cardiovascular deaths and a 1.4-fold increase in myocardial infarctions (MIs) at 2 years compared to nondiabetic patients [5]. Multiple factors, such as hyperglycemia, endo- thelial dysfunction, and oxidative stress, play a vital role in platelet hyperreactivity in diabetic patients. As such, the higher thrombotic risk in patients with ACS and diabetes highlights the need for adequate antithrombotic protection [6].

Inhibition of platelet aggregation with dual antiplatelet therapy (DAPT) consisting of low-dose aspirin and a $\mathrm{P}_{2} \mathrm{Y}_{12}$ receptor inhibitor is recognized as a standard treatment for patients after ACS. An impaired response to clopidogrel that occurs in $5 \%$ to $44 \%$ of patients with diabetes has been reported in multiple pharmacodynamic studies [7]. Prasugrel and ticagrelor, third-generation $\mathrm{P}_{2} \mathrm{Y}_{12}$ inhibitors, circumvent the clinical limitations of clopidogrel, such as liver metabolism, drug interactions, and polymorphisms in genes encoding platelet receptors, thereby exerting faster and stronger antiplatelet aggregation properties, which suggests their usefulness in patients with ACS and diabetes [8, 9]. Existing guidelines recommend that ACS patients use 
ticagrelor or prasugrel instead of clopidogrel if there is no contraindication $[10,11]$; however, real-world registration data showed that clopidogrel is still widely used [12, 13], which may be, in part, attributable to the higher bleeding risk associated with more potent antithrombosis.

Ticagrelor has been demonstrated to reduce the composite of ischemic events without increasing the overall risk of major bleeding compared with clopidogrel in ACS patients [9]. However, most of the data came from randomized controlled studies in Western countries, and the effectiveness and safety of ticagrelor in East Asian populations have not yet been fully established. The "East Asian Paradox" means that East Asian patients have a lower risk of ischemic events but a higher risk of bleeding complications than non-East Asian patients, despite lower responsiveness to antiplatelet therapy $[14,15]$, suggesting that Asian patients may not have a better benefit-risk ratio after using more potent $\mathrm{P}_{2} \mathrm{Y}_{12}$ inhibitors (such as ticagrelor). Therefore, we aimed to compare the 6-month clinical outcomes between ticagrelor and clopidogrel in patients with ACS and diabetes and hopefully provide valuable data in an Asian population.

\section{Methods}

2.1. Study Design and Population. The study was a singlecenter, prospective, randomized, open-label, blinded endpoint, and controlled registry trial carried out at the Institute of Cardiovascular Medicine, the Second Affiliated Hospital, Army Medical University in China. We consecutively enrolled 270 patients with ACS and diabetes. For the study patients, ACS was determined based on the diagnosis of unstable angina or acute MI. Unstable angina pectoris was defined as a patient with symptoms of myocardial ischemia but no increase in troponin, with or without ischemic changes in the electrocardiogram, such as ST-segment depression or new $\mathrm{T}$ wave inversion. Acute MI was defined as ST elevation MI or non-ST elevation MI. Each patient underwent percutaneous coronary intervention in the study. Type 2 diabetes was defined as individuals with fasting blood glucose $\geq 126 \mathrm{mg} / \mathrm{dL}(7.0 \mathrm{mmol} / \mathrm{L})$ or random blood glucose $\geq 200 \mathrm{mg} / \mathrm{dL}(11.1 \mathrm{mmol} / \mathrm{L})$ or patients with a known history of diabetes who were undergoing hypoglycemic therapy. Fasting was defined as no calorie intake for $\geq 8$ hours. The main exclusion criteria were any contraindications to antiplatelet drugs, the need for oral anticoagulation therapy, the simultaneous use of potent inhibitors or inducers of cytochrome P450 3A, and the combination of chronic infections, malignant tumors, and autoimmune diseases.

This randomized controlled trial is aimed at evaluating the effectiveness and safety of two different antiplatelet strategies. The protocol (Supplemental Figure 1) was approved by the Institutional Ethics Committee, and the trial was registered at http://www.chictr.org.cn (ChiCTR1800015104). The study was conducted in accordance with the Declaration of Helsinki following the Good Clinical Practice Guidelines. Fifty-seven patients refused to enter after reading the informed consent form, and ultimately, informed consent forms from 270 eligible participants were obtained. This report complied with the Consolidated Standards of Reporting Trial (CONSORT) statement.

2.2. Randomization and Treatment Groups. Eligible patients were randomly assigned to the ticagrelor group or the clopidogrel group at a $1: 1$ ratio through an interactive voice response or network response system. Randomization codes were generated in blocks of constant size. Randomization was carried out, and once a patient was included, administration of the study regimen started. The treatment groups were allocated in an open-label manner. Patients in the ticagrelor group received a loading dose of $180 \mathrm{mg}$, followed by oral ticagrelor at $90 \mathrm{mg}$, taken twice per day, while patients in the clopidogrel group who had not received a loading dose and had not taken clopidogrel for at least 5 days before randomization received a loading dose of $300 \mathrm{mg}$, followed by a dosage of $75 \mathrm{mg}$ per day, or a maintenance dosage of $75 \mathrm{mg}$ per day. During the entire study period, all patients received oral aspirin at $100 \mathrm{mg}$ once per day.

2.3. Data Collection. Data including the patients' baseline characteristics, past medical history, risk factors, clinical diagnosis, medications at the time of admission and discharge, in-hospital biochemistry, and interventions/procedures were collected from questionnaires by a specially trained staff worker. Percutaneous coronary intervention (PCI) was performed in a conventional manner. All patients were given antiplatelet drugs before the intervention, with aspirin and clopidogrel or ticagrelor, according to the principle of randomization.

2.4. Follow-Up and Clinical Outcomes. Follow-up was performed for 6 months by phone interview or personal contact, and data on efficacy (nonfatal MI, target vessel revascularization, rehospitalization, stroke, and death from any cause) and safety (bleeding events) outcomes were collected. MI was defined according to the fourth universal definition proposed in 2018. Target vessel revascularization was defined as percutaneous revascularization or bypass surgery for the target lesion or any arterial segment containing the target lesion. Rehospitalization was defined as hospitalization for unstable angina. Ischemic stroke was characterized by the onset of neurological dysfunction caused by focal brain, spinal cord, or retinal infarction. The Bleeding Academic Research Alliance (BARC) standards were used to evaluate bleeding events.

2.5. Statistical Analysis. Continuous variables are represented by the median (interquartile range, IQR), and categorical variables are represented by $n(\%)$. As appropriate, the Mann-Whitney $U$ test, Pearson's $\chi^{2}$ test, the continuity correction test, or Fisher's exact test was used to compare the differences between the ticagrelor group and the clopidogrel group. Binary logistic regression models were employed to identify the independent risk factors. Cox proportional hazards regression analysis was used to explore the correlation between the different antiplatelet regimens and clinical outcomes. Variables considered to be clinically relevant or that showed statistically univariate significance with clinical outcomes $(p<0.20)$ were included in the multivariate 
regression model. To ensure the simplicity of the final model, the variables were carefully selected based on the number of available events, and co-linearity was avoided. The odds ratio (OR) or hazard ratio (HR) was used to clarify the relations between risk factors and clinical outcomes. $p<0.05$ was considered statistically significant. Statistical analyses were performed using SPSS software version 20 (IBM Corp., Armonk, NY, USA). Statistical power calculations were performed using PASS software, version 11 (NCSS, LLC, Kaysville, UT, USA).

\section{Results}

3.1. Patients. A total of 270 ACS patients with diabetes were enrolled in the current study between October 2017 and March 2019. The 6-month follow-up period ended in September 2019. The recruited patients were randomly divided into the clopidogrel group $(n=135)$ and the ticagrelor group $(n=135)$. At 6 months, $266(98.5 \%)$ of the 270 patients had complete follow-up data available, and 4 patients ( 2 in the ticagrelor group and 2 in the clopidogrel group) were lost to follow-up due to missing phone numbers or their own reasons (Supplemental Figure 1). Both the clopidogrel group $(n=133)$ and the ticagrelor group $(n=133)$ were well balanced in almost all baseline characteristics (Table 1), including demographics, medical history, medication, biomedical indicators, and the results of coronary angiography, although patients with hypertension were more likely to be in the clopidogrel group $(p=0.038)$. There seemed to be more patients in the ticagrelor group with chronic kidney disease, but the difference was not significant $(p=0.053)$.

3.2. Clinical Outcomes. At 6 months, the proportion of successful revascularizations in the ticagrelor group was lower than that in the clopidogrel group in terms of efficacy outcomes, but there was no significant difference between the two groups $(14.3 \%$ vs. $16.5 \%, p=0.610)$. For the safety outcome, the total number of bleeding events defined by BARC in the ticagrelor group was slightly more than that in the clopidogrel group, although there was no significant difference ( $24.1 \%$ vs. $15.8 \%, p=0.091)$; particularly in the BARC type 2 group, the bleeding risk in the ticagrelor group showed a tendency to increase $(6.0 \%$ vs. $1.5 \%, p=0.053)$ (Table 2$)$.

3.3. Risk Factors of Outcomes. The demographic characteristics, medical history, medication, biomedical indicators, the results of coronary angiography, and grouping were included in the univariate logistic regression model analysis, and age, hypertension, liver insufficiency, hemoglobin, and estimated glomerular filtration rate (eGFR) were potential influencing factors for the composite effectiveness endpoint (Supplemental Table 1). Then, through the multivariate model for calibration analysis, we found that liver insufficiency was an independent risk factor that affected the effectiveness outcomes $(p=0.006)$ (Table 3$)$. The same logistic regression model was used to analyze the possible risk factors for the bleeding endpoints (Table 4 and Supplemental Table 2).
3.4. Survival Analysis. Univariate and multivariate Cox proportional hazards regression models were used serially to identify the factors affecting the survival outcome of the efficacy and safety endpoints, and the included variables were the same as those mentioned above. Age, hypertension, liver insufficiency, hemoglobin, and eGFR were possible confounding factors for the survival outcome of the effectiveness endpoints (Supplemental Table 1), and liver insufficiency $(p=0.002)$ and eGFR $(p=0.026)$ were found to be independent factors influencing the survival of the effectiveness endpoints (Table 3) in the multivariate model. Through the same statistical model, the treatment grouping was demonstrated to be an independent factor that affected the survival outcome of the bleeding endpoints defined by BARC (Table 4 and Supplemental Table 2).

Based on the Cox survival regression analysis model, we further compared the differences in the 6-month follow-up endpoint events between the two treatment groups. The results showed that there was no significant difference in the survival rate of the effectiveness endpoint between the two groups (HR 0.83, 95\% CI 0.44-1.56, $p=0.561$ ) (Figure 1), but the incidence of bleeding events in the ticagrelor group was higher than that in the clopidogrel group (HR 1.76, 95\% CI 1.00-3.10, $p=0.049$ ) (Figure 2).

\section{Discussion}

The study was conducted to compare the 6-month clinical outcomes between the clopidogrel and ticagrelor groups in Asian patients with ACS and diabetes. The main findings of our study on a Chinese population were that ticagrelor did not improve the survival rate of efficacy outcomes (composite of nonfatal MI, target vessel revascularization, rehospitalization, stroke, and death from any cause) but increased the prevalence of bleeding events defined by BARC criteria in patients with ACS and diabetes compared to clopidogrel.

Diabetes has a clear negative impact on the clinical outcome of ACS patients [16]. Although the underlying causes may be multifaceted $[17,18]$, platelet insufficiency is common in diabetic patients, in whom hyperglycemia, endothelial and vascular damage, and chronic proinflammatory and prothrombotic environments promote platelet activation $[19,20]$. Highly reactive platelets are a key factor that accelerates atherosclerosis and leads to adverse ischemic or thrombotic events $[6,21]$. Therefore, the strength of the antiplatelet regimen is very important for patients with ACS and diabetes [22]. The "East Asian Paradox" refers to the low potential risk of ischemic events, but the high risk of bleeding in East Asian populations, which poses a challenge to the current "one size fits all" antiplatelet therapy strategy for ACS patients [23-25]. In dealing with the specific population of patients with ACS combined with diabetes, it is necessary to pay attention to the more complex balance between ischemia and bleeding complications and further optimize the antiplatelet strategy, which is conducive to improving patient outcomes.

At present, the results of studies on optimized dual antithrombotic regimens for patients with ACS and diabetes are 
TABLE 1: Baseline characteristics of ACS patients with diabetes.

\begin{tabular}{|c|c|c|c|c|}
\hline & Total $(n=266)$ & Ticagrelor plus aspirin $(n=133)$ & Clopidogrel plus aspirin $(n=133)$ & $p$ value \\
\hline Age, years & $64.0(57.0-69.0)$ & $64.0(56.0-68.0)$ & $64.0(57.0-69.0)$ & 0.671 \\
\hline Males, $n(\%)$ & $86(32.3 \%)$ & $39(29.3 \%)$ & $47(35.3 \%)$ & 0.294 \\
\hline BMI, $\mathrm{kg} / \mathrm{m}^{2}$ & $24.8(22.9-27.3)$ & $24.8(23.0-27.6)$ & $24.8(22.8-26.8)$ & 0.404 \\
\hline Current smoker, $n(\%)$ & $141(53.0 \%)$ & $73(54.9 \%)$ & $68(51.1 \%)$ & 0.539 \\
\hline Current drinking, $n(\%)$ & $107(40.2 \%)$ & $52(39.1 \%)$ & $55(41.4 \%)$ & 0.708 \\
\hline $\mathrm{UAP}, n(\%)$ & $199(74.8 \%)$ & $96(72.2 \%)$ & $103(77.4 \%)$ & 0.323 \\
\hline STEMI, $n(\%)$ & $32(12.0 \%)$ & $19(14.3 \%)$ & $13(9.8 \%)$ & 0.258 \\
\hline NSTEMI, $n(\%)$ & $35(13.2 \%)$ & $18(13.5 \%)$ & $17(12.8 \%)$ & 0.856 \\
\hline Heart rate, bpm & $78.0(70.0-87.0)$ & $78.0(71.0-88.0)$ & $78.0(68.5-86.0)$ & 0.402 \\
\hline $\mathrm{SBP}, \mathrm{mmHg}$ & $131.5(117.0-144.3)$ & $132.0(115.0-149.5)$ & $131.0(118.0-142.0)$ & 0.682 \\
\hline $\mathrm{DBP}, \mathrm{mmHg}$ & $73.0(63.0-82.0)$ & $73.0(61.5-82.0)$ & $73.0(65.0-81.5)$ & 0.687 \\
\hline \multicolumn{5}{|l|}{ History } \\
\hline Previous MI, $n$ (\%) & $34(12.8 \%)$ & $21(15.8 \%)$ & $12(9.8 \%)$ & 0.142 \\
\hline $\begin{array}{l}\text { Previous coronary stent } \\
\text { implantation, } n(\%)\end{array}$ & $46(17.3 \%)$ & $26(19.5 \%)$ & $20(15.0 \%)$ & 0.331 \\
\hline Previous GI bleeding, $n(\%)$ & $8(3.0 \%)$ & $4(3.0 \%)$ & $4(3.0 \%)$ & 1.000 \\
\hline Hypertension, $n$ (\%) & $176(66.2 \%)$ & $80(60.2 \%)$ & $96(72.2 \%)$ & 0.038 \\
\hline Hyperuricemia, $n$ (\%) & $15(5.6 \%)$ & $6(4.5 \%)$ & $9(6.8 \%)$ & 0.425 \\
\hline Hyperlipemia, $n$ (\%) & $57(21.4 \%)$ & $28(21.1 \%)$ & $29(21.8 \%)$ & 0.881 \\
\hline Liver insufficiency, $n(\%)$ & $11(4.1 \%)$ & $8(63.0 \%)$ & $3(2.3 \%)$ & 0.124 \\
\hline Chronic kidney disease, $n(\%)$ & $30(11.3 \%)$ & $20(15.0 \%)$ & $10(7.5 \%)$ & 0.053 \\
\hline Ischemic stroke, $n(\%)$ & $22(8.3 \%)$ & $9(6.8 \%)$ & $13(9.8 \%)$ & 0.373 \\
\hline \multicolumn{5}{|l|}{ Medication } \\
\hline Statins, $n(\%)$ & $262(98.5 \%)$ & $132(99.2 \%)$ & $130(97.7 \%)$ & 0.314 \\
\hline Nitrate, $n(\%)$ & $66(24.8 \%)$ & $32(24.1 \%)$ & $34(25.6 \%)$ & 0.776 \\
\hline Beta blockers, $n(\%)$ & $198(74.7 \%)$ & $97(72.9 \%)$ & $101(76.5 \%)$ & 0.502 \\
\hline RAAS inhibitors, $n(\%)$ & $192(72.5 \%)$ & $91(68.9 \%)$ & $101(75.9 \%)$ & 0.202 \\
\hline Calcium channel blockers, $n(\%)$ & $71(26.7 \%)$ & $33(24.8 \%)$ & $38(28.6 \%)$ & 0.488 \\
\hline Proton pump inhibitors, $n(\%)$ & $148(55.6 \%)$ & $76(57.2 \%)$ & $72(54.1 \%)$ & 0.622 \\
\hline Insulin, $n(\%)$ & $95(35.7 \%)$ & $52(39.1 \%)$ & $43(32.3 \%)$ & 0.249 \\
\hline Metformin, $n(\%)$ & $120(45.1 \%)$ & $57(42.9 \%)$ & $63(47.4 \%)$ & 0.460 \\
\hline Acarbose, $n(\%)$ & $74(27.8 \%)$ & $33(24.8 \%)$ & $41(30.8 \%)$ & 0.274 \\
\hline Other hypoglycemic agents, $n(\%)$ & $97(36.5 \%)$ & $42(31.6 \%)$ & $55(41.4 \%)$ & 0.098 \\
\hline \multicolumn{5}{|l|}{ Biomedical indicators } \\
\hline Leukocyte, $10^{9} / \mathrm{L}$ & $6.9(5.9-8.2)$ & $7.0(5.9-8.3)$ & $6.8(5.9-8.1)$ & 0.534 \\
\hline Hemoglobin, g/L & $130.0(118.0-142.0)$ & $131.0(116.5-143.0)$ & $129.0(119.0-141.5)$ & 0.802 \\
\hline Platelets, $10^{9} / \mathrm{L}$ & $189.5(150.0-220.3)$ & $189.0(155.0-224.0)$ & $188.0(146.5-219.0)$ & 0.597 \\
\hline Mean platelet volume, fL & $11.5(10.4-12.6)$ & $11.4(10.4-12.5)$ & $11.6(10.4-12.6)$ & 0.413 \\
\hline Platelet distribution width, fL & $16.0(13.8-17.1)$ & $15.5(13.7-17.0)$ & $16.2(14.0-17.3)$ & 0.124 \\
\hline ALT, U/L & $23.7(17.1-36.3)$ & $23.7(17.4-37.5)$ & $23.7(16.8-36.3)$ & 0.773 \\
\hline AST, U/L & $21.1(16.1-28.3)$ & $21.1(16.3-29.2)$ & $20.6(15.6-26.1)$ & 0.369 \\
\hline Creatinine, $\mu \mathrm{mol} / \mathrm{L}$ & $76.2(63.0-88.4)$ & $77.3(65.0-88.7)$ & $74.5(61.3-88.4)$ & 0.317 \\
\hline eGFR, $\mathrm{mL} / \mathrm{min}$ & $88.0(73.0-96.0)$ & $88.0(74.5-95.0)$ & $88.0(73.0-97.0)$ & 0.906 \\
\hline Total cholesterol, mmol/L & $3.7(3.1-4.5)$ & $3.7(3.1-4.5)$ & $3.7(3.1-4.5)$ & 0.437 \\
\hline Triglyceride, $\mathrm{mmol} / \mathrm{L}$ & $1.5(1.1-2.1)$ & $1.4(1.0-1.9)$ & $1.5(1.2-2.1)$ & 0.085 \\
\hline Glycosylated hemoglobin, \% & $7.7(6.8-8.9)$ & $7.7(6.8-9.2)$ & $7.7(6.8-8.7)$ & 0.565 \\
\hline
\end{tabular}


TABLE 1: Continued.

\begin{tabular}{lccc}
\hline & Total $(n=266)$ & Ticagrelor plus aspirin $(n=133)$ & Clopidogrel plus aspirin $(n=133)$ \\
\hline Coronary angiography & & & \\
Single-vessel disease, $n(\%)$ & $42(15.8 \%)$ & $18(13.5 \%)$ & $24(18.0 \%)$ \\
Double-vessel disease, $n(\%)$ & $105(39.5 \%)$ & $48(36.1 \%)$ & $57(42.9 \%)$ \\
Triple-vessel disease, $n(\%)$ & $119(44.7 \%)$ & $67(50.4 \%)$ & $52(39.1 \%)$ \\
\hline
\end{tabular}

Data were expressed as $n$ (\%) and median (IQR). IQR: interquartile range; $p$ value, Mann-Whitney $U$ test, or Pearson chi-square test; ACS: acute coronary syndrome; ALT: alanine aminotransferase; AST: aspartate aminotransferase; BMI: body mass index; DBP: diastolic blood pressure; eGFR: estimated glomerular filtration rate; GI: gastrointestinal; MI: myocardial infarction; NSTEMI: non-ST-segment elevation myocardial infarction; STEMI: ST-segment elevation myocardial infarction; SBP: systolic blood pressure; RAAS: renin-angiotensin-aldosterone system; UAP: unstable angina pectoris.

TABLE 2: Clinical outcomes in ACS patients with diabetes.

\begin{tabular}{|c|c|c|c|c|}
\hline & Total $(n=266)$ & Ticagrelor plus aspirin $(n=133)$ & Clopidogrel plus aspirin $(n=133)$ & $p$ value \\
\hline \multicolumn{5}{|l|}{ Efficacy outcome } \\
\hline Composite endpoint & $41(15.4 \%)$ & $19(14.3 \%)$ & $22(16.5 \%)$ & 0.610 \\
\hline Nonfatal MI & $0(0.0 \%)$ & $0(0.0 \%)$ & $0(0.0 \%)$ & - \\
\hline Target vessel revascularization & $15(5.6 \%)$ & $5(3.8 \%)$ & $10(7.5 \%)$ & 0.184 \\
\hline Rehospitalization & $17(6.4 \%)$ & $9(6.8 \%)$ & $8(6.0 \%)$ & 0.802 \\
\hline Ischemic stroke & $3(1.1 \%)$ & $1(0.8 \%)$ & $2(1.5 \%)$ & 1.000 \\
\hline Death from any cause & $6(2.3 \%)$ & $4(3.0 \%)$ & $2(1.5 \%)$ & 0.680 \\
\hline \multicolumn{5}{|l|}{ BARC type } \\
\hline BARC $1 \sim 5$ & $53(19.9 \%)$ & $32(24.1 \%)$ & $21(15.8 \%)$ & 0.091 \\
\hline BARC 1 & $38(14.3 \%)$ & $21(15.8 \%)$ & $17(12.8 \%)$ & 0.483 \\
\hline BARC 2 & $10(3.8 \%)$ & $8(6.0 \%)$ & $2(1.5 \%)$ & 0.053 \\
\hline BARC 3 & $4(1.5 \%)$ & $2(1.5 \%)$ & $2(1.5 \%)$ & 1.000 \\
\hline BARC 4 & $0(3.5 \%)$ & $0(0.0 \%)$ & $0(0.0 \%)$ & - \\
\hline BARC 5 & $1(0.4 \%)$ & $1(0.8 \%)$ & $0(0.0 \%)$ & 1.000 \\
\hline
\end{tabular}

Data were expressed as $n$ (\%) and median (IQR). IQR: interquartile range; $p$ value, Pearson chi-square test, continuity correction test, or Fisher's exact test; composite endpoints included MI, revascularization, rehospitalization for angina, stroke, and death from any cause; BARC: Bleeding Academic Research Consortium definition for bleeding; MI: myocardial infarction.

TABLE 3: Risk factors for the composite efficacy outcomes of ACS patients with diabetes in multivariable analysis.

\begin{tabular}{|c|c|c|c|c|}
\hline Variable & Multivariable OR (95\% CI) & $p 1$ value & Multivariable OR (95\% CI) & $p 2$ value \\
\hline Age, years & $1.04(0.98-1.09)$ & 0.186 & $1.03(0.98-1.08)$ & 0.267 \\
\hline \multicolumn{5}{|l|}{ History } \\
\hline Hypertension & $2.14(0.90-5.09)$ & 0.085 & $1.85(0.84-4.05)$ & 0.125 \\
\hline Liver insufficiency & $6.55(1.73-24.78)$ & 0.006 & $4.52(1.74-11.77)$ & 0.002 \\
\hline \multicolumn{5}{|l|}{ Biomedical indicator } \\
\hline Hemoglobin & $0.99(0.98-1.01)$ & 0.184 & $0.99(0.98-1.00)$ & 0.181 \\
\hline eGFR & $0.98(0.97-1.00)$ & 0.069 & $0.98(0.97-1.00)$ & 0.026 \\
\hline Grouping (ticagrelor vs. clopidogrel) & - & - & $0.83(0.44-1.56)$ & 0.561 \\
\hline
\end{tabular}

95\% CI: 95\% confidence interval; OR: odds ratio; $p 1$ : logistic regression analysis; $p 2$ : Cox survival analysis; BMI: body mass index; MI: myocardial infarction; GI: gastrointestinal; RAAS: renin-angiotensin-aldosterone system; ALT: alanine aminotransferase; eGFR: estimated glomerular filtration rate.

controversial. The PLATO study shows that compared with clopidogrel, ticagrelor treatment significantly reduced the risk of major adverse cardiovascular events (MACEs) in patients with ACS and played an effective role in antithrombosis without significantly increasing the risk of major bleeding [26]. A substudy of PLATO showed that ticagrelor showed a better benefit-risk value than clopidogrel regard- less of diabetes status and blood sugar control [9]. In the subgroup analysis of the TRITON-TIMI 38 trial, prasugrel, another effective ADP P2Y12 antagonist, reduced the risk of cardiovascular death, myocardial infarction, or stroke by $4.8 \%$ compared with clopidogrel (30\% relative) [8].

However, some studies have different conclusions. Spoendlin et al. conducted a cohort study using United 
TABLE 4: Risk factors for bleeding events defined by the BARC criteria in ACS patients with diabetes in multivariable analysis.

\begin{tabular}{|c|c|c|c|c|}
\hline Variable & Multivariable OR (95\% CI) & $p$ value & Multivariable OR (95\% CI) & $p$ value \\
\hline Age, years & $0.97(0.93-1.00)$ & 0.056 & $0.97(0.94-1.00)$ & 0.068 \\
\hline \multicolumn{5}{|l|}{ History } \\
\hline Chronic kidney disease & $0.37(0.11-1.29)$ & 0.120 & $0.39(0.12-1.26)$ & 0.117 \\
\hline \multicolumn{5}{|l|}{ Biomedical indicator } \\
\hline Triglyceride & $1.13(0.94-1.35)$ & 0.204 & $1.11(0.98-1.27)$ & 0.107 \\
\hline Grouping (ticagrelor vs. clopidogrel) & $1.80(0.95-3.41)$ & 0.070 & $1.76(1.00-3.10)$ & 0.049 \\
\hline
\end{tabular}

95\% CI: 95\% confidence interval; OR: odds ratio; $p 1$ : logistic regression analysis; $p 2$ : Cox survival analysis; BMI: body mass index; MI: myocardial infarction; GI: gastrointestinal; RAAS: renin-angiotensin-aldosterone system; ALT: alanine aminotransferase; eGFR: estimated glomerular filtration rate.

HR 0.83 95\% CI: $0.44-1.56$

$P=0.561$

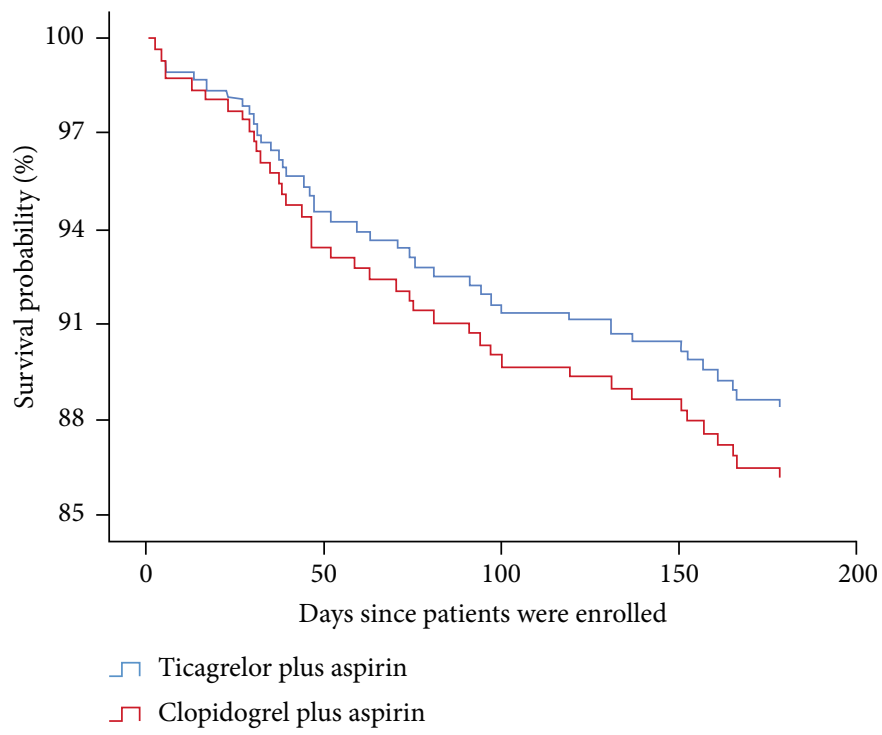

FIGURE 1: Event-free survival for the composite of efficacy outcomes in ACS patients with diabetes. There was no significant difference in the survival outcomes of MACEs between the ticagrelor group (blue line) and the clopidogrel group (red line) (HR 0.83, 95\% CI 0.44-1.56, $p=0.561)$.

States commercial claims data (2009-2015) in ACS patients with diabetes and revealed that the prasugrel group had better cardiovascular outcomes despite a higher risk of shortterm bleeding. No significant difference was found in two different comparisons between the ticagrelor and clopidogrel groups or between the prasugrel and ticagrelor groups [27]. Data from the Korean Acute MI Registry-National Institutes of Health showed that in MI patients with diabetes undergoing PCI, the use of prasugrel/ticagrelor $(n=1000)$ did not improve the composite of cardiac death, recurrent $\mathrm{MI}$, or stroke but significantly increased the number of major bleeding events compared with clopidogrel treatment $(n=2985)$ [28]. Additionally, our research has obtained a similar result in that ticagrelor increased the incidence of bleeding events without improving the efficacy outcomes, suggesting that East Asian patients may potentially be different from Western patients. Furthermore, Goto et al. performed a study on the optimized antiplatelet regimen of ACS patients with diabetes in Japan, Taiwan, and South
Korea and found that the number of major bleeding events in the ticagrelor treatment group was higher, albeit not significantly. However, there was no significant difference in ischemia risk between the ticagrelor and clopidogrel groups [29]. Park et al. compared the treatment differences between ticagrelor and clopidogrel in Korean acute myocardial infarction (AMI) patients, $22 \%$ of whom had diabetes, and found that ticagrelor did not reduce the risk of ischemia but increased the 6-month Thrombolysis in Myocardial Infarction (TIMI) major bleeding events [30].

Furthermore, in the current study, age, hypertension, liver insufficiency, hemoglobin, and eGFR were found to be potential influencing factors for the composite effectiveness endpoint in patients with ACS and diabetes in univariate logistic regression model analysis, and liver insufficiency was an independent risk factor that affected the effectiveness outcomes in the multivariate model for calibration analysis. In addition, there are many factors that may affect the prognosis of patients who underwent PCI, including their own 
HR 1.76 95\% CI: $1.00-3.10$

$P=0.049$

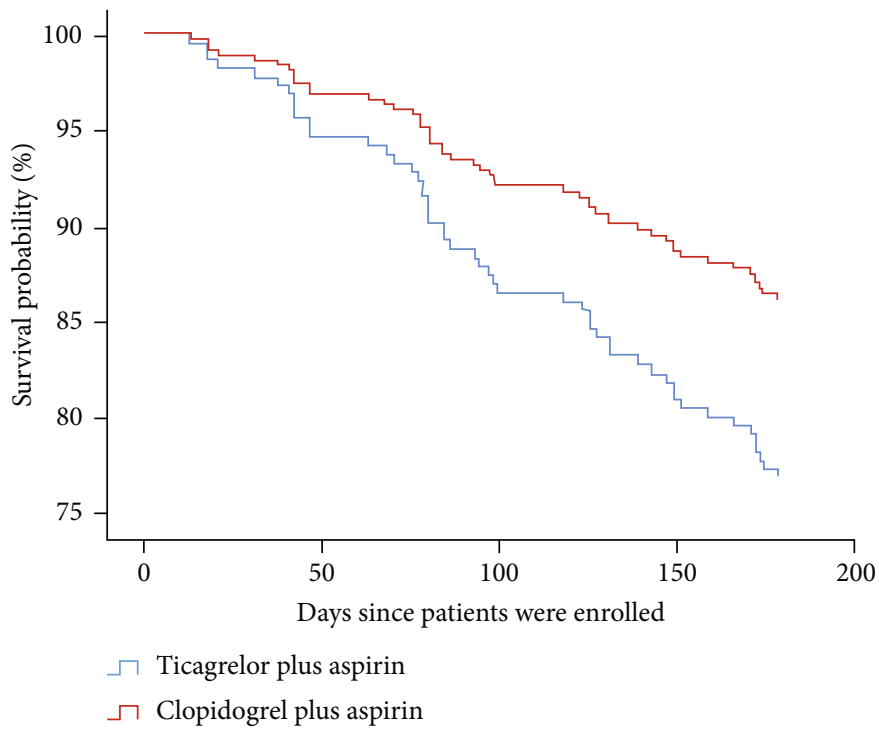

FIgURE 2: Event-free survival for bleeding events defined by the BARC criteria in ACS patients with diabetes. The incidence of bleeding events in the ticagrelor group (red line) was higher than that in the clopidogrel group (blue line) (HR 1.76, 95\% CI 1.00-3.10, $p=0.049$ ).

conditions, the types and specifications of implanted devices, and the choices and timing of medications. A meta-analysis of 64 randomized controlled trials and 102735 patients after approximately 20 months of follow-up showed that the type of stent implanted seems to have a partial impact on the risk of adverse events in patients and that different treatment durations of DAPT are also related to bleeding risk [31].

Atherothrombosis is a highly complex process [32], and a considerable amount of data shows that ethnic differences have an impact on thrombosis, which is reflected by coagulation, fibrinolysis, and inflammation markers [33]. East Asian patients have a low body mass index (BMI) and are significantly different from Western patients in terms of thrombosis, platelet receptor inhibition, and susceptibility to bleeding risk. Therefore, East Asian patients have a higher risk of bleeding associated with antiplatelet therapy during antithrombotic therapy [23-25]. This may explain why our results did not reveal a difference in effectiveness between the two groups but showed an increased risk of bleeding in the ticagrelor group compared to the clopidogrel group. Most of the current trials evaluating the clinical efficacy and safety of $\mathrm{P} 2 \mathrm{Y} 12$ receptor potent inhibitors (ticagrelor/prasugrel) in ACS patients with diabetes do not include a sufficient number of East Asian participants, and it is difficult to draw trustworthy conclusions [15]. Therefore, before using the powerful P2Y12 inhibitors recommended by studies conducted on Western populations to treat patients with ACS complicated with diabetes, more specific studies on East Asian populations in this field are required.

This study has several limitations. First, although our study is based on prospective, randomized, open-label, blinded endpoints, and controlled registries, it is a smallscale, single-center study, and the small sample size may limit the power to detect differences in clinical outcomes.
Second, we did not include information on the lifestyle of the patients regarding the type of diet and frequency of exercise per week or the frequencies of drinking and smoking. This lack of information seems slightly rudimentary in terms of lifestyle surveys. Third, middle-aged and elderly heart disease patients usually have other diseases, such as diabetes, hypertension, and gout, which causes them to take multiple drug treatments. Actually, the impact of polypharmacy with the varied disease backgrounds and other complications the patients have made it difficult to arrive at a definitive conclusion of the study. Fourth, the duration of follow-up was limited, and it is possible that a longer follow-up period could have displayed significantly different outcomes between the ticagrelor and clopidogrel groups of ACS patients with diabetes.

\section{Conclusion}

Our study shows that ticagrelor did not improve the composite of nonfatal MI, target vessel revascularization, rehospitalization, stroke, and death from any cause; however, ticagrelor significantly increased the number of bleeding events defined by the BARC criteria in Chinese patients with ACS and diabetes during the 6-month follow-up compared with clopidogrel. These results seem to suggest the need to transform antiplatelet strategies for the treatment of ACS patients with diabetes from "one guideline suitable for all races" to "racially differentiated antiplatelet therapy," but more dedicated studies in East Asian populations are required.

\section{Data Availability}

The data that support the findings of this study are available from the corresponding author upon reasonable request. 


\section{Conflicts of Interest}

The authors declare that they have no conflicts of interest.

\section{Authors' Contributions}

JJ and YH designed the study. PH, XL, and YL acquired data. $\mathrm{JL}, \mathrm{XW}$, and LH analyzed and interpreted the data. JL wrote the manuscript. All authors reviewed/edited the manuscript. All authors read and approved the final manuscript. $\mathrm{PH}$ and $\mathrm{XL}$ contributed equally to this work. Peixun $\mathrm{He}$ and Xiaolin Luo are co-first authors.

\section{Acknowledgments}

This work was supported by grants from the National Key Research and Development Program (2016YFC1301300 and 2016YFC1301304). The authors thank Renzheng Chen, MS, for helping develop the statistical design and perform statistical analyses.

\section{Supplementary Materials}

Supplemental Figure 1: flow chart of the study. Supplemental Table 1: risk factors for the composite efficacy outcomes of ACS patients with diabetes in univariable analysis. Supplemental Table 2: risk factors for the bleeding events defined with BARC criteria of ACS patients with diabetes in univariable analysis. (Supplementary Materials)

\section{References}

[1] F. O. Alenazy and M. R. Thomas, "Novel antiplatelet targets in the treatment of acute coronary syndromes," Platelets, vol. 32, no. 1, pp. 15-28, 2021.

[2] Z. M. Ruggeri, "Platelets in atherothrombosis," Nature Medicine, vol. 8, no. 11, pp. 1227-1234, 2002.

[3] L. Wang, H. L. Cong, J. X. Zhang et al., “Triglyceride-glucose index predicts adverse cardiovascular events in patients with diabetes and acute coronary syndrome," Cardiovascular Diabetology, vol. 19, no. 1, p. 80, 2020.

[4] K. K. Sharma, M. Mathur, S. Lodha, S. K. Sharma, N. Sharma, and R. Gupta, "Study of differences in presentation, risk factors and management in diabetic and nondiabetic patients with acute coronary syndrome," Indian Journal of Endocrinology and Metabolism, vol. 20, no. 3, pp. 354-358, 2016.

[5] K. Malmberg, S. Yusuf, H. C. Gerstein et al., "Impact of diabetes on long-term prognosis in patients with unstable angina and non-Q-wave myocardial Infarction," Circulation, vol. 102, no. 9, pp. 1014-1019, 2000.

[6] J. F. Arthur, K. Jandeleit-Dahm, and R. K. Andrews, "Platelet hyperreactivity in diabetes: focus on GPVI signaling-are useful drugs already available?," Diabetes, vol. 66 , no. 1 , pp. 7-13, 2017.

[7] M. Karaźniewicz-Łada, D. Danielak, P. Burchardt et al., "Clinical pharmacokinetics of clopidogrel and its metabolites in patients with cardiovascular diseases," Clinical Pharmacokinetics, vol. 53, no. 2, pp. 155-164, 2014.

[8] S. D. Wiviott, E. Braunwald, D. J. Angiolillo et al., "Greater clinical benefit of more intensive oral antiplatelet therapy with prasugrel in patients with diabetes mellitus in the trial to assess improvement in therapeutic outcomes by optimizing platelet inhibition with prasugrel-thrombolysis in myocardial infarction 38," Circulation, vol. 118, no. 16, pp. 1626-1636, 2008.

[9] S. James, D. J. Angiolillo, J. H. Cornel et al., "Ticagrelor vs. clopidogrel in patients with acute coronary syndromes and diabetes: a substudy from the PLATelet inhibition and patient outcomes (PLATO) trial," European Heart Journal, vol. 31, no. 24, pp. 3006-3016, 2010.

[10] M. Roffi, C. Patrono, J. P. Collet et al., "2015 ESC guidelines for the management of acute coronary syndromes in patients presenting without persistent ST-segment elevation," European Heart Journal, vol. 37, no. 3, pp. 267-315, 2016.

[11] M. Valgimigli, H. Bueno, R. A. Byrne et al., "2017 ESC focused update on dual antiplatelet therapy in coronary artery disease developed in collaboration with EACTS," European Heart Journal, vol. 39, no. 3, pp. 213-260, 2018.

[12] U. Khalid, S. Bandeali, P. G. Jones et al., "Prescription patterns of clopidogrel, prasugrel, and ticagrelor after percutaneous coronary intervention with stent implantation (from the NCDR PINNACLE registry)," The American Journal of Cardiology, vol. 124, no. 12, pp. 1807-1812, 2019.

[13] C. Larmore, M. B. Effron, C. Molife et al., "“Real-World" comparison of prasugrel with ticagrelor in patients with acute coronary syndrome treated with percutaneous coronary intervention in the United States," Catheterization and Cardiovascular Interventions, vol. 88, no. 4, pp. 535-544, 2016.

[14] J. Kang and H. S. Kim, "The evolving concept of dual antiplatelet therapy after percutaneous coronary intervention: focus on unique feature of east Asian and "Asian paradox"," Korean Circulation Journal, vol. 48, no. 7, pp. 537-551, 2018.

[15] Y. H. Jeong, “"East Asian paradox": challenge for the current antiplatelet strategy of "one-guideline-fits-all races" in acute coronary syndrome," Current Cardiology Reports, vol. 16, no. 5 , p. $485,2014$.

[16] K. Franklin, R. J. Goldberg, F. Spencer et al., "Implications of diabetes in patients with acute coronary syndromes: the global registry of acute coronary events," Archives of Internal Medicine, vol. 164, no. 13, pp. 1457-1463, 2004.

[17] A. Avogaro, M. Albiero, L. Menegazzo, S. de Kreutzenberg, and G. P. Fadini, "Endothelial dysfunction in diabetes: the role of reparatory mechanisms," Diabetes Care, vol. 34, Supplement 2, pp. S285-S290, 2011.

[18] G. de Luca, M. Verdoia, S. Savonitto et al., "Impact of diabetes on clinical outcome among elderly patients with acute coronary syndrome treated with percutaneous coronary intervention," Journal of Cardiovascular Medicine, vol. 21, no. 6, pp. 453-459, 2020.

[19] D. Calderone, D. Capodanno, and D. J. Angiolillo, “An updated drug profile of ticagrelor with considerations on the treatment of patients with coronary artery disease and diabetes mellitus," Expert Review of Cardiovascular Therapy, vol. 18, no. 8, pp. 449-464, 2020.

[20] D. L. Bhatt, J. W. Eikelboom, S. J. Connolly et al., "Role of combination antiplatelet and anticoagulation therapy in diabetes mellitus and cardiovascular Disease," Circulation, vol. 141, no. 23, pp. 1841-1854, 2020.

[21] S. Lindemann, B. Krämer, K. Daub, K. Stellos, and M. Gawaz, "Molecular pathways used by platelets to initiate and accelerate atherogenesis," Current Opinion in Lipidology, vol. 18, no. 5, pp. 566-573, 2007.

[22] S. S. Sethi, E. G. Akl, and M. E. Farkouh, "Diabetes mellitus and acute coronary syndrome: lessons from randomized 
clinical trials," Current Diabetes Reports, vol. 12, no. 3, pp. 294-304, 2012.

[23] Y. Huo, Y.-H. Jeong, Y. Gong et al., "2018 update of expert consensus statement on antiplatelet therapy in east Asian patients with ACS or undergoing PCI," Scientific Bulletin, vol. 64, no. 3, pp. 166-179, 2019.

[24] G. N. Levine, Y. H. Jeong, S. Goto et al., "World Heart Federation expert consensus statement on antiplatelet therapy in East Asian patients with ACS or undergoing PCI," Nature Reviews Cardiology, vol. 11, no. 10, pp. 597-606, 2014.

[25] G. N. Levine, Y. H. Jeong, S. Goto et al., "World heart federation expert consensus statement on antiplatelet therapy in east asian patients with ACS or undergoing PCI," Global Heart, vol. 9, no. 4, p. 457, 2020.

[26] L. Wallentin, R. C. Becker, A. Budaj et al., "Ticagrelor versus clopidogrel in patients with acute coronary syndromes," The New England Journal of Medicine, vol. 361, no. 11, pp. 10451057, 2009.

[27] J. Spoendlin, J. J. Gagne, J. J. Lewey, E. Patorno, S. Schneeweiss, and R. J. Desai, "Comparative effectiveness and safety of antiplatelet drugs in patients with diabetes mellitus and acute coronary syndrome," Pharmacoepidemiology and Drug Safety, vol. 27, no. 12, pp. 1361-1370, 2018.

[28] K. T. Ahn, S. W. Seong, U. L. Choi et al., "Comparison of 1-year clinical outcomes between prasugrel and ticagrelor versus clopidogrel in type 2 diabetes patients with acute myocardial infarction underwent successful percutaneous coronary intervention," Medicine, vol. 98, no. 11, article e14833, 2019.

[29] S. Goto, C. H. Huang, S. J. Park, H. Emanuelsson, and T. Kimura, "Ticagrelor vs. clopidogrel in Japanese, Korean and Taiwanese patients with acute coronary syndrome - randomized, double-blind, phase III PHILO study," Circulation Journal, vol. 79, no. 11, pp. 2452-2460, 2015.

[30] D. W. Park, O. Kwon, J. S. Jang et al., "Clinically significant bleeding with ticagrelor versus clopidogrel in Korean patients with acute coronary syndromes intended for invasive Management," Circulation, vol. 140, no. 23, pp. 1865-1877, 2019.

[31] F. D’Ascenzo, M. Iannaccone, G. Saint-Hilary et al., "Impact of design of coronary stents and length of dual antiplatelet therapies on ischaemic and bleeding events: a network metaanalysis of 64 randomized controlled trials and 102735 patients," European Heart Journal, vol. 38, no. 42, pp. 31603172, 2017.

[32] G. Davì and C. Patrono, "Platelet activation and atherothrombosis," The New England Journal of Medicine, vol. 357, no. 24, pp. 2482-2494, 2007.

[33] P. A. Gurbel, K. P. Bliden, E. Cohen et al., "Race and sex differences in thrombogenicity: risk of ischemic events following coronary stenting," Blood Coagulation \& Fibrinolysis, vol. 19, no. 4, pp. 268-275, 2008. 